\title{
TMEM45B is a novel predictive biomarker for prostate cancer progression and metastasis
}

\author{
F. $\mathrm{LUO}^{1,2,3}, \mathrm{~K} . \mathrm{YANG}^{4}$, Y. Z. WANG ${ }^{1,2}$, D. $\mathrm{LIN}^{1,2, *}$ \\ ${ }^{1}$ The Vancouver Prostate Centre, Vancouver General Hospital, Vancouver, BC, Canada; ${ }^{2}$ Department of Experimental Therapeutics, BC Cancer \\ Agency, Vancouver, BC, Canada; ${ }^{3}$ Department of Urology, Tianjin Union Medical Center, Tianjin, China; ${ }^{4}$ Department of Urology, The Second \\ Hospital of Tianjin Medical University, Tianjin Institute of Urology, Tianjin, China
}

${ }^{*}$ Correspondence: dlin@bccrc.ca

Received August 22, 2017 / Accepted February 28, 2018

\begin{abstract}
It is urgently required to explore the clinical relevance of TMEM45B expression and Prostate cancer (PCa), and determine the predictive significance of TMEM45B as a biomarker for PCa patients. Patient-derived xenograft (PDX) models were developed for PCa with different metastatic potential (LTL-418, LTL-313B, LTL-313H and LTL-331). The gene expression microarray of LTL-313H and LTL-313B which derived from a single PCa patient was performed to identify the candidate biomarker gene, TMRM45B. MSKCC and TCGA PCa patient cohorts were introduced to analyze the clinical significance of TMEM45B expression. Quantitative Real-Time PCR (qRT-PCR) revealed that there was a significant increase of TMEM45B expression in the LTL-313H and LTL-331 high metastatic potential tumor lines compared to the LTL-418, LTL-313B low metastatic potential tumor lines. In the MSKCC PCa cohort, the mRNA level of TMEM45B in patients with metastasis was significantly higher than that in primary $\mathrm{PCa}(\mathrm{p}=0.001)$ and benign prostate hyperplasia patients $(\mathrm{BPH})(\mathrm{p}<0.001)$. In addition, the increased TMEM45B expression positively related with higher possibility of biochemical recurrence $(\mathrm{p}=0.016)$, distant metastasis $(\mathrm{p}<0.001)$ and overall patient survival $(\mathrm{p}=0.07)$. Moreover, TMEM45B expression was considered an independent risk factor for metastasis of PCa based on multivariate logistic regression; where KaplanMeier analysis showed that patients with elevated TMEM45B had a shorter biochemical recurrence free survival (RFS). In addition, the primary PCa patient subgroup analysis revealed significant association between TMEM45B expression and clinical features, where cases with elevated TMEM45B were more likely to develop metastasis compared to those without it in the N0 primary PCa patient cohort ( $\mathrm{p}=0.010)$ (Table 3). The primary PCa patient cohort TCGA was used to validate the results, and an obvious relationship was found between TMEM45B and PCa clinical characteristic (T/N stage, Gleason score, Recurrence/Progression). Furthermore, a significant poor disease free survival (DFS) was established in high-level TMEM45B patients compared to those with low levels $(\mathrm{p}=0.007)$. The combined results indicate that increased TMEM45B expression appears significantly associated with prostate carcinoma progression and metastasis, and this provides a new prognostic biomarker for predicting metastatic potential in PCa patients; especially those with primary PCa.
\end{abstract}

Key words: TMEM45B, prognosis, biomarker, prostate cancer, metastasis, patient-derived xenograft

With an estimated 161,360 newly diagnosed patients and 26,730 deaths in the United States in 2017 [1], prostate cancer (PCa) has a significant impact on society and is a major clinical challenge. In China, PCa had a great increase in incidence due to increasing westernized lifestyle, despite the gradual implementation of prostate-specific antigen (PSA) screening and improved biopsy techniques. It is estimated that approximately 60,300 cases were newly diagnosed as PCa there, and that 26,600 would die from $\mathrm{PCa}$ in 2015. Localized PCa is highly manageable using curative treatment such as definitive radical prostatectomy
(RC) or radiotherapy techniques, but it is estimated that up to a fifth of patients experience recurrence after curative treatment [2]. Although first-generation androgen deprivation therapies are widely used in advanced or recurred $\mathrm{PCa}$, the disease progresses after a varied quiescent period into castrate-resistant PCa (CRPC) and metastatic PCa; and this is considered both inevitable and incurable [3]. Therefore, it is urgently required to discover new aggressiveness/ metastasis-related biomarkers for patient stratification and earlier effective treatment to improve the oncological and survival outcomes. 
PCa metastasis require a complex series of biological changes and it is also affected by a variety of risk factors [4]. One of the obstacles to studying cancer metastasis is cancer heterogeneity. To overcome this hurdle we transplanted tumor tissues derived from different biopsy sites of a single PCa patient into the subrenal capsule site of NOD-SCID mice and developed multiple patient-derived xenograft (PDX) models with different metastatic ability (LTL-313 series model) [5]. The xenograft tumor lines preserve the characteristics of their parental tumors and have varying metastatic ability in vivo, so they therefore provide a valuable tool for studying the underlying PCa metastasis mechanisms and discovering novel predictive biomarkers $[5,6]$.

Herein, we identified TMEM45B as a potential candidate biomarker gene associated with PCa metastasis by comparing gene expression analysis in the high-LTL$313 \mathrm{H}$ metastatic potential PDX model and the LTL-313B low-metastatic potential model. TMEM45B is a member of the trans-membrane proteins gene family (TMEM) which encodes different cellular membrane proteins $[7,8]$. While varied TMEM protein expression has been observed in the following cancers; breast $[9,10]$, renal [11], ovarian [12], lung [13] and liver [9], the function of the TMEM proteins, especially TMEM45B, in PCa remains unclear. This current study investigated the expression levels of TMEM45B in multiple PCa tumor/cell lines and validated its correlation with clinical biological behavior. This demonstrated TMEM45B's potential for predicting aggression/metastasis in primary PCa patients.

\section{Materials and methods}

Chemicals, solvents and solutions were obtained from Sigma-Aldrich (Oakville, ON, Canada) and human LNCaP, C4-2, 22Rv-1 and VCaP PCa cell lines were obtained from the American Type Culture Collection (ATCC).

Development of PDX models. PCa tumor tissues were cut into small cubes $\left(3\right.$ by 3 by $1 \mathrm{~mm}^{3}$ ). These were then grafted under the kidney capsule of male NOD/SCID mice. The development of PDX models was as previously described [5], and the mice were bred in a specific pathogen-free (SPF) level facility in the Animal Resource Center (ARC) at the BC Cancer Research Centre. All animal operation followed the Canadian Council guidelines for Animal Care.

Histopathology and Immunohistochemistry. Preparation of paraffin-embedded tissue sections and routine $\mathrm{H} \& \mathrm{E}$ staining were performed as previously described [5]. Immunohistochemical (IHC) staining was according to manufacture protocol and the following rabbit polyclonal antibodies were used for immunohistochemistry; anti-AR: ABR, ant-PSA: Dako and anti-PTEN Cell Signaling Technology.

Gene expression microarray and data analysis. The gene expression microarray dataset of the LTL-313H vs LTL-313B xenograft tumor lines was normalized with Z-score trans- formation. The candidate genes were chosen according to the gene expression difference of the low tumor lines, the fold change was set at FC>2.0 and there were 566 candidates left. The microarray dataset of the two tumor lines was then compared by t-test to determine the significant overexpressed candidates; and 81 genes showed significantly differential expression of LTL313H and LTL313B $(p<0.05)$. Our literature search focused on candidates not previously reported in prostate cancer. Although the TMEM protein family is reported to have an important role in cancer carcinogenesis and metastasis in several tumor types, to the best of our knowledge it has not been studied in prostate cancer. Therefore, we focused on TMEM45B, and the gene expression microarray dataset is available on GEO: GSE41193.

Total RNA isolation and quantitative real-time PCR (qRT-PCR). Total RNA was isolated from cultured cells and tissues by TRIzol and qRT-PCR was performed with ViiA 7 Real-Time PCR system (Applied Biosystems, Foster City, CA). The TMEM45B primer sequences were: Forward primer, TTGGAACACCCGAATGGGAC, Reverse primer, GCATCTCGGCTCATTCCTCA. Reference gene GAPDH primer sequences were: Forward primer, CACCAGGGCTGC TTTTAA CTC, Reverse primer, GACAAGCTTCCCGTTCTCAG.

Clinical relevance and prognosis analysis. MSKCC [14] and TCGA [15] clinical data were obtained from the CBio Cancer Genomics Portal website, and the expression values of TMEM45B are presented in $\log 2$ median-centered intensity [16]. The association of TMEM45B expression and clinical parameters in PCa patient cohort was assessed by Student t-test. Binary logistic regression assessed risk factors for PCa metastasis and PCa patient survival outcome were analyzed by Kaplan-Meier and the log-rank test. The significant threshold level was $\mathrm{p}<0.05$.

\section{Results}

H\&E and IHC staining of low-metastatic LTL-313B and high-metastatic LTL-313H. H\&E and IHC staining visualized the pathological features of the single-patient deprived PDX model (LTL-313B and LTL-313H). H\&E staining demonstrated similar histopathological features in both models and similar results were found for IHC staining of AR, PSA and PTEN in the two tumor lines. This is consistent with the same-patient origin of the models. Interestingly, PCa-xenograft tumor lung metastasis was observed in LTL-313H, but no metastases lesions were found in the LTL-313B tumor line (Figure 1).

Increased TMEM45B mRNA expression in metastatic PDX models and cell lines. The candidate gene was chosen because of the difference in LTL-313H/LTL-313B gene microarray in the tumor samples available on GEO: GSE41193 [5]. The normalized TMEM45B gene expression of LTL-313H and LTL-313B xenograft tumor lines is shown in Figure 2. RT-PCR investigated the TMEM45B expression of LTL-418, 


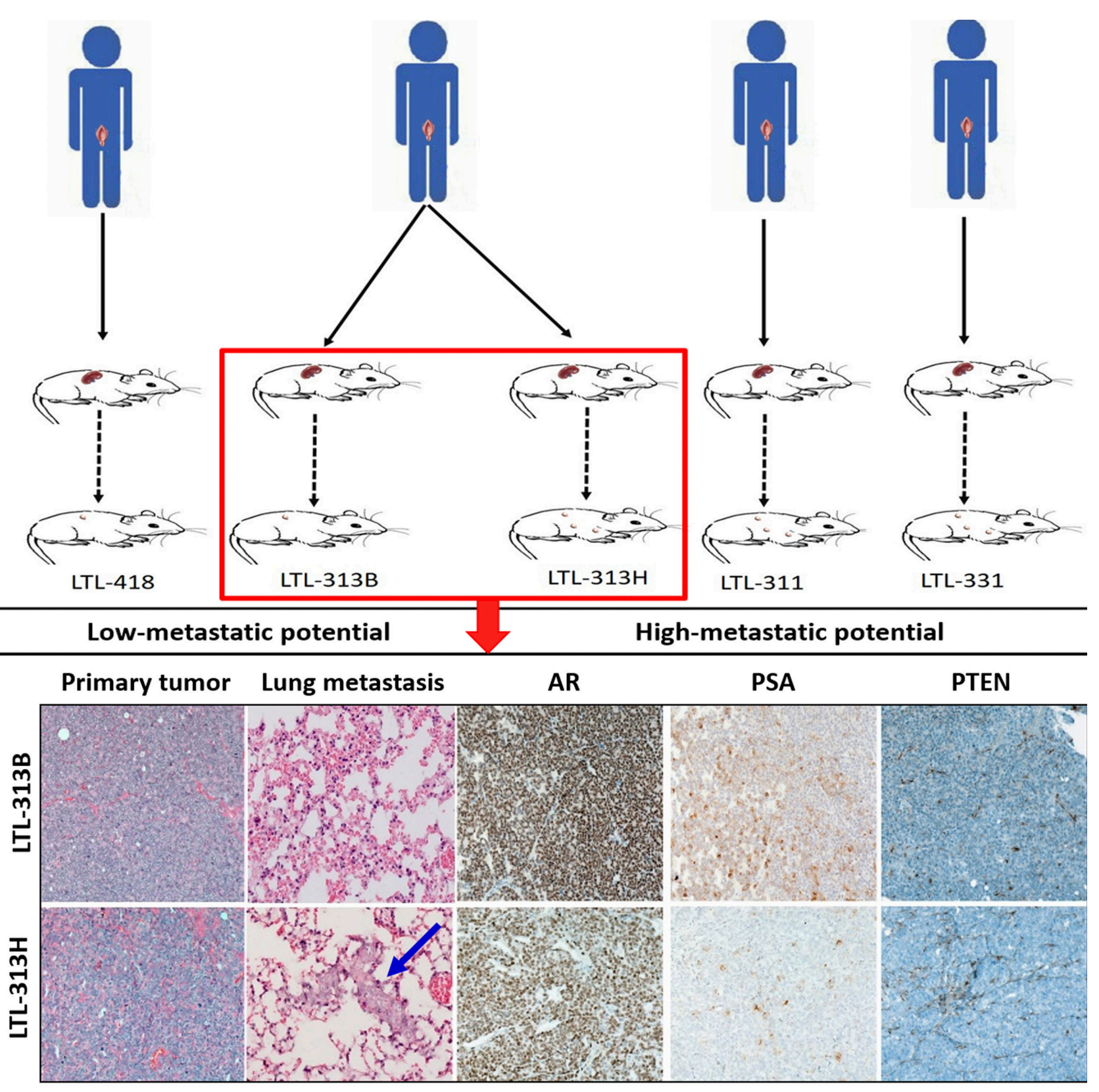

Figure 1. PDX model diagram and H\&E/IHC staining of LTL-313B and LTL-313H $(200 \times)$

LTL-313B, LTL-313H and LTL-331 tumor lines, revealing significant over-expression of TMEM45B in the LTL-313H, LTL-331 high-potential metastatic tumor lines compared to the LTL-418, LTL-313B low-potential metastatic lines (Figure $3 \mathrm{~A}$ ). In addition, the TMEM45B mRNA level of highinvasive/metastatic potential cell lines (VCaP, C4-2, 22RV-1) was also higher than that in the LNCaP cell lines (Figure 3B).

Correlation of TMEM45B expression and PCa progress/ metastasis. To further validate the clinical relevance of TMEM45B expression and tumor biological behavior, the MSKCC PCa patient cohort was introduced to compare the
TMEM45B expression level in benign prostate hyperplasia $(\mathrm{BPH})$ and primary and metastatic PCa. Figure 3 highlights that TMEM45B expression in metastasis tumor was significantly higher than in $\mathrm{BPH}(\mathrm{p}<0.001)$ and primary $\mathrm{PCa}$ $(\mathrm{p}=0.001)$ (Figure 4).

Comparative analysis determined the correlation of TMEM45B expression and clinical characteristics, and this revealed that TMEM45B expression was increased in patients with PSA levels $>10 \mathrm{ng} / \mathrm{ml}(\mathrm{p}=0.034)$, $\mathrm{pT}$ stage $\geq \mathrm{T} 3 \mathrm{~A}$ $(\mathrm{p}=0.011)$ and Gleason score $\geq 8(\mathrm{p}=0.001)$ (Table 1$)$. In addition, the prognosis outcomes assessment showed signifi- 
TMEM45B

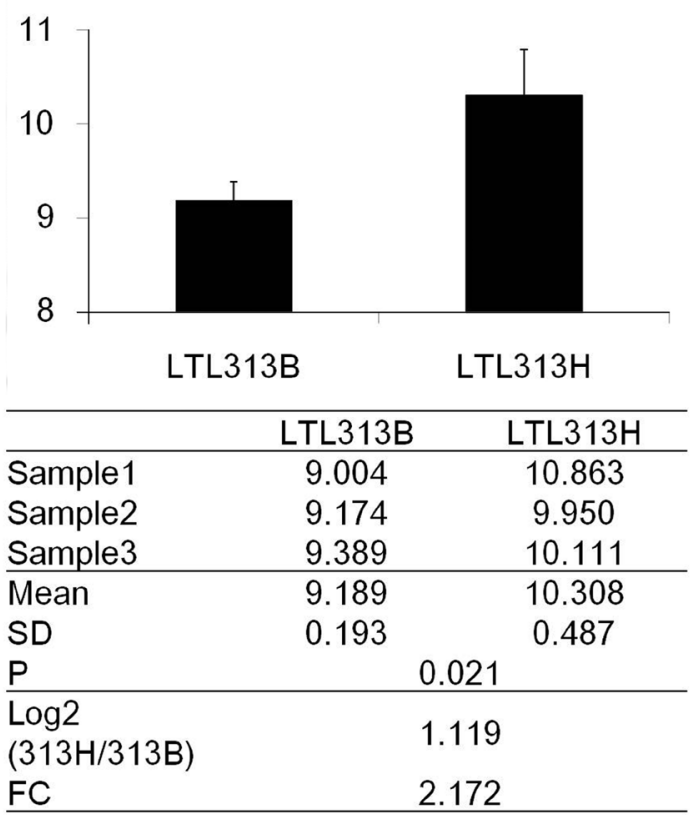

Figure 2. TMEM45B expression of the xenograft tumor lines and cell lines.

Table 1. The association of TMEM45B expression and clinical characteristics of PCa in MSKCC.

\begin{tabular}{|c|c|c|c|c|}
\hline Variable & Value & Mean & SD & p-value \\
\hline \multirow{2}{*}{ PSA } & $\leq 10$ & 8.60 & 0.78 & \multirow{2}{*}{$0.034^{*}$} \\
\hline & $>10$ & 8.97 & 1.03 & \\
\hline \multirow{2}{*}{ pT stage } & $\leq \mathrm{T} 2 \mathrm{C}$ & 8.51 & 0.71 & \multirow{2}{*}{$0.011^{\star}$} \\
\hline & $\geq \mathrm{T} 3 \mathrm{~A}$ & 8.85 & 0.86 & \\
\hline \multirow{2}{*}{ Gleason Score } & $\leq 7$ & 8.53 & 0.73 & \multirow{2}{*}{$0.001^{*}$} \\
\hline & $\geq 8$ & 9.15 & 0.92 & \\
\hline \multirow{2}{*}{ BCR } & positive & 8.89 & 0.79 & \multirow{2}{*}{$0.016^{*}$} \\
\hline & negative & 8.54 & 0.89 & \\
\hline \multirow{2}{*}{ Met } & $\mathrm{Y}$ & 9.38 & 1.02 & \multirow{2}{*}{$<0.001^{*}$} \\
\hline & $\mathrm{N}$ & 8.56 & 0.76 & \\
\hline \multirow{2}{*}{ Death } & $\mathrm{Y}$ & 9.22 & 1.01 & \multirow{2}{*}{$0.007^{\star}$} \\
\hline & $\mathrm{N}$ & 8.64 & 0.83 & \\
\hline
\end{tabular}

pT stage: pathological stage BCR: biochemical recurrence, Met: metastasis Gleason score: five distinct glands growth patterns of PCa were described to predict the tumor behavior, PCa with a low Gleason score are less likely to show aggressive behavior. cant association between TMEM45B and biochemical recurrence $(p=0.016)$, distant metastasis $(p<0.001)$ and overall mortality $(\mathrm{p}=0.07)$ (Table 1$)$.

Finally, univariate and multivariate logistic regression established that Gleason score $(\mathrm{OR}=4.81$, 95\% CI $=1.16-$ 19.95, $\mathrm{p}=0.030)$ and TMEM45B expression $(\mathrm{OR}=2.16$, 95\% $\mathrm{CI}=1.05-4.45, \mathrm{p}=0.037$ ) were independent risk factors for metastasis of PCa in the MSKCC cohort (Table 2), and Kaplan-Meier analysis determined that TMEM45B was significantly associated with biochemical recurrencefree survival (defined as the PSA level rebounding, RFS; Figure 5A; $\mathrm{p}=0.009$ ).

Relevant TMEM45B expression and primary PCa clinical features. Subgroup analysis of primary PCa patients investigated the association of TMEM45B and clinical characteristics in the primary patient cohort; confirming that patients with $\mathrm{PSA}>10 \mathrm{ng} / \mathrm{ml}$, Gleason score $\geq 8$ and $\mathrm{T}$ stage $\geq \mathrm{T} 3 \mathrm{~A}$ had higher TMEM45B level than other patient groups. Furthermore, patients with elevated TMEM45B were more likely to develop metastasis than those without it in the N0 primary PCa patient cohort $(p=0.010)$ (Table 3). Patients with elevated TMEM45B also had a significantly poor biochemical recurrence-free survival than those in the control group $(\mathrm{p}=0.027)$ (Figure 5B).

The TCGA primary PCa cohort further validated that the relevant TMEM45B expression level was significantly different between the non-aggressive-tumor patients $(\mathrm{T} \leq 2 \mathrm{C}$, Gleason $\leq 7)$ and the aggressive-tumor group $(\mathrm{T} \geq 3 \mathrm{~A}$, Gleason $\geq 8$ ) (Table 4). Patients with high TMEM45 levels were more likely to develop lymph node metastasis $(\mathrm{p}=0.017)$ (Table 4), and increased TMEM45 was associated with poor disease-free survival (DSF) in the primary PCa cohort (Table 3, Figure 5C).

\section{Discussion}

$\mathrm{PCa}$ is a heterogeneous disease because some prostate carcinomas present indolent clinical behavior as low-malignant tumors and others can possess the aggressive phenotype for fatal malignancies. Although prognosis-related clinical indicators such as Gleason score, TNM stage and prostatespecific antigen (PSA) levels have been performed for practical decision making, confusion remains in the assess-

Table 2. Univariate and Multivariate analyses of PCa metastasis in MSKCC.

\begin{tabular}{|c|c|c|c|c|c|c|c|c|}
\hline & \multicolumn{4}{|c|}{ Univariate } & \multicolumn{4}{|c|}{ Multivariate } \\
\hline & \multirow{2}{*}{ OR } & \multicolumn{2}{|c|}{$95 \% \mathrm{CI}$} & \multirow{2}{*}{ p-value } & \multirow{2}{*}{ OR } & \multicolumn{2}{|c|}{$95 \% \mathrm{CI}$} & \multirow{2}{*}{ p-value } \\
\hline & & Lower & Upper & & & Lower & Upper & \\
\hline Age & 1.04 & 0.99 & 1.08 & 0.121 & & & & \\
\hline PSA & 1.03 & 1.01 & 1.06 & 0.012 & 1.01 & 1.00 & 1.03 & 0.054 \\
\hline pT stage* & 5.38 & 1.81 & 16.02 & 0.003 & 68.21 & 0.50 & 9298.04 & 0.092 \\
\hline Gleason score ${ }^{\#}$ & 14.35 & 5.04 & 40.85 & 0.000 & 4.81 & 1.16 & 19.95 & $0.030^{*}$ \\
\hline TMEM45B & 2.84 & 1.72 & 4.69 & 0.000 & 2.16 & 1.05 & 4.45 & $0.037^{*}$ \\
\hline
\end{tabular}

${ }^{*} \mathrm{pT}$ stage was divided into two groups $(\mathrm{pT} \geq \mathrm{T} 2 \mathrm{C}$ vs $\mathrm{pT} \leq \mathrm{T} 2 \mathrm{~B}){ }^{*}$ Gleason score was re-grouped as $\mathrm{G}>8$ vs $\mathrm{G} \leq 8$. 
A

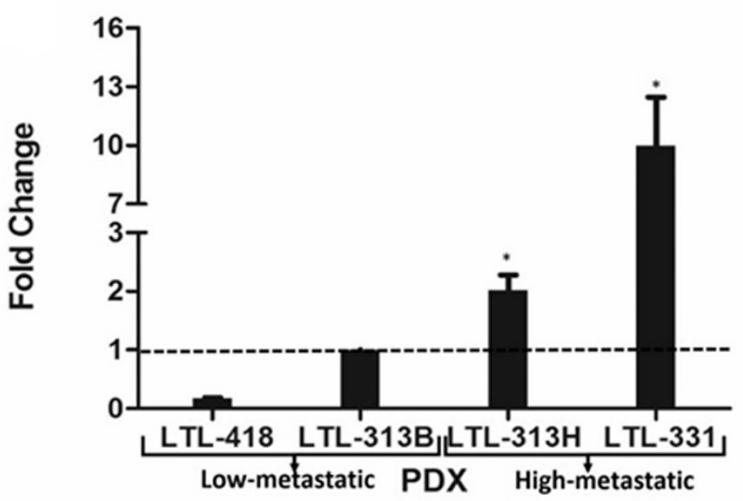

B

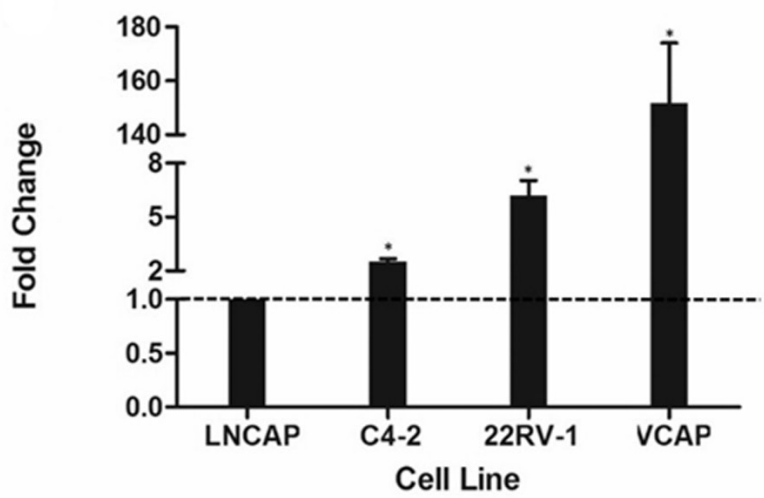

Figure 3. The normalized gene expression of TMEM45B of the two tumor lines.

ment of treatment options for the different PCa patients. It is therefore necessary to identify novel and efficient diagnostic and prognostic biomarkers for more reliable prediction of $\mathrm{PCa}$; expecially for primary PCa.

Herein, we analyzed the microarray data of paired PDX models (LTL-313H and LTL-313B) and identified TMEM45B as a candidate associated with PCa aggressiveness and metastasis. We demonstrated that elevated TMEM45B expression is significantly associated with aggressive status. In addition, PCa patients with increased TMEM45B had lower recurrence-free survival time and higher metastasis possibility, and this indicates that TMEM45B expression is an independent risk factor for PCa metastasis. Most importantly, the primary PCa patient cohort with higher TMEM45B expression had aggressive phenotype, metastatic potential and poor RFS/DFS outcome; and these suggest that TMEM45B is a strong predictive biomarker for primary $\mathrm{PCa}$.

TMEM proteins are reported to participate in many biological processes and in a variety of normal and tumor tissues. For example, 7 TMEM variants were found in kidney tumor tissues (TMEM213, TMEM61, TMEM30B, TMEM116, TMEM45B, TMEM22 and TMEM45A) [11]. Researchers have also observed significant deregulation of 10 TMEM variants in renal cell carcinoma (RCC), thus indicating the importance of the TMEM family in this pathogenesis. TMEM45B, which was first found in thermal aggregation in SDS-PAGE gels, has been demonstrated to be a valuable predictor for many malignancies [7]. In addition to the T. Wrzesiński et al. report that TMEM45B played an important role in the pathogenesis of RCC [11], TMEM45B is also reported to have a significant prognostic and predictive value in pathologic staging and histology grade and is therefore a standard clinical molecular marker in breast cancer [17]. Similarly, Zhao's study illustrated that TMEM45B expression was significantly up-regulated and positively correlated with the cell cycle and migration pathways. This important role of TMEM45B was

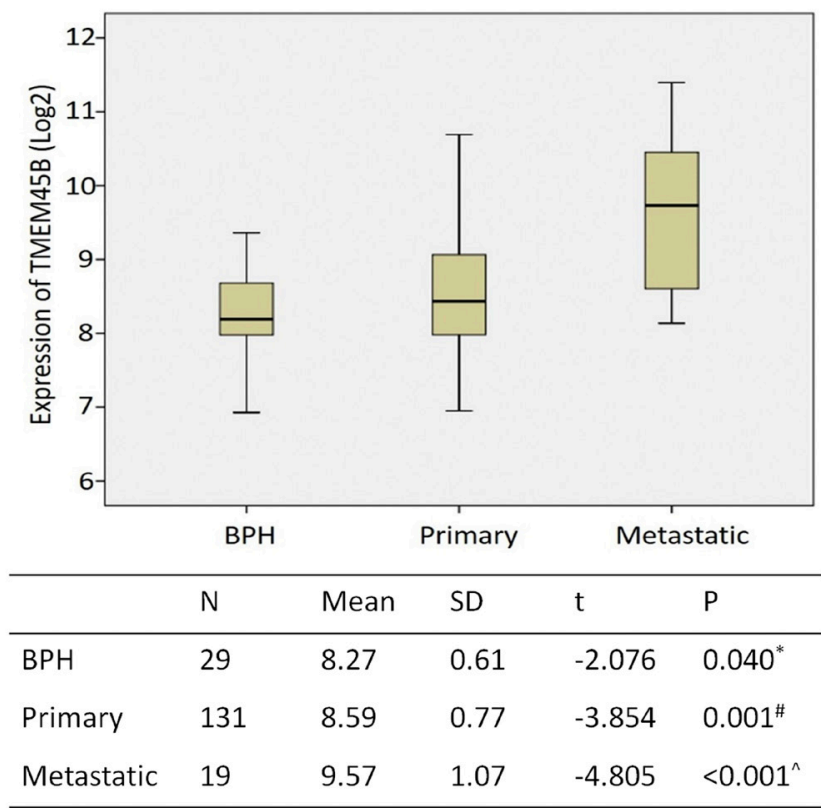

Figure 4. Expression of TMEM45B in BPH, primary and metastasis PCa patients. ${ }^{\star} \mathrm{BPH}$ vs primary, \#Primary vs Metastatic, $\wedge \mathrm{BPH}$ vs Metastatic

Table 3. The association of TMEM45B expression and clinical characteristics of Primary PCa in MSKCC.

\begin{tabular}{|c|c|c|c|c|c|}
\hline & Variable & Value & Mean & SD & p-value \\
\hline \multirow{6}{*}{$\begin{array}{l}\text { TMEM45B } \\
\text { expression of } \\
\text { primary PCa }\end{array}$} & \multirow{2}{*}{ PSA } & $\leq 10$ & 8.51 & 0.74 & \multirow{2}{*}{$0.040^{*}$} \\
\hline & & $>10$ & 8.84 & 0.83 & \\
\hline & \multirow{2}{*}{ pT stage } & $\leq \mathrm{T} 2 \mathrm{C}$ & 8.49 & 0.70 & \multirow{2}{*}{$0.040^{*}$} \\
\hline & & $\geq \mathrm{T} 3 \mathrm{~A}$ & 8.78 & 0.86 & \\
\hline & \multirow{2}{*}{$\begin{array}{l}\text { Gleason } \\
\text { Score }\end{array}$} & $\leq 7$ & 8.52 & 0.72 & \multirow{2}{*}{$0.007^{\star}$} \\
\hline & & $\geq 8$ & 9.08 & 0.94 & \\
\hline \multirow{2}{*}{$\begin{array}{l}\text { TMEM45B } \\
\text { expression of } \\
\text { N0 primary PCa }\end{array}$} & \multirow[b]{2}{*}{ Met } & $\mathrm{Y}$ & 9.26 & 0.73 & \multirow[b]{2}{*}{0.010} \\
\hline & & $\mathrm{N}$ & 8.57 & 0.77 & \\
\hline
\end{tabular}



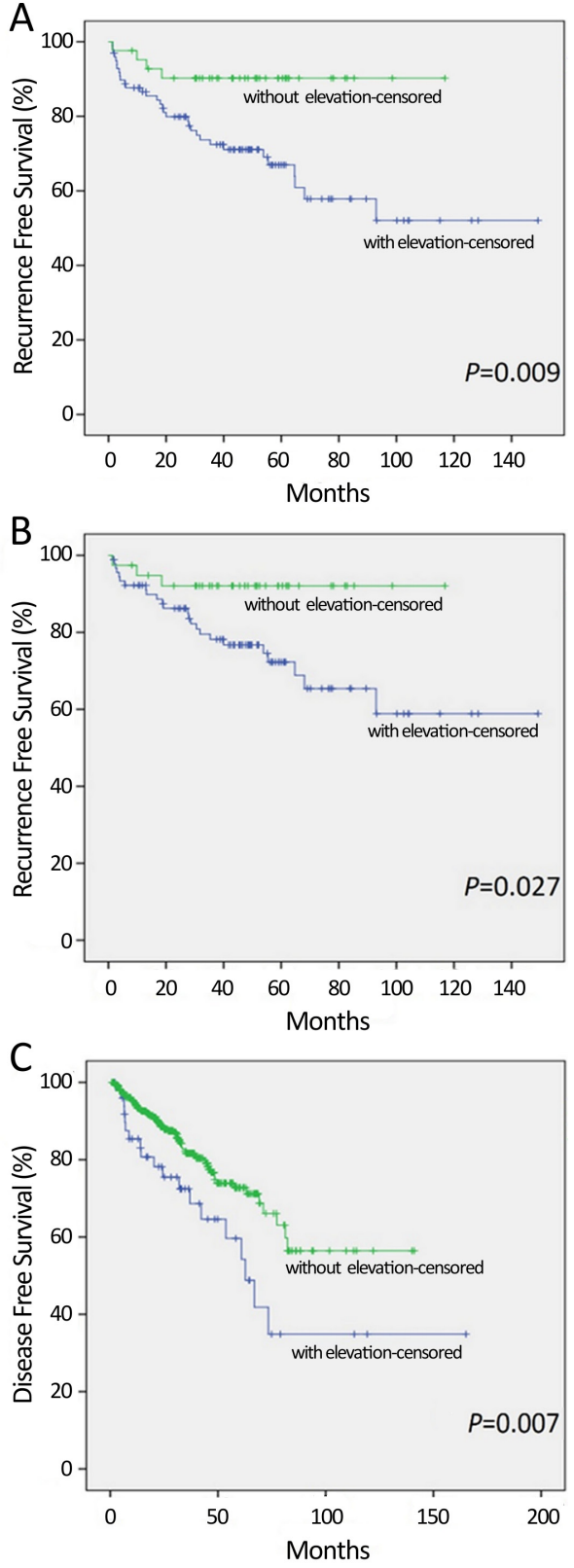

Figure 5. Survival analysis: A) Kaplan-Meier analysis of biochemical RFS of all PCa patients in MSKCC, B) Kaplan-Meier analysis of biochemical RFS among Primary patients in MSKCC cohort and C) Kaplan-Meier analysis of DFS among primary PCa patients in TCGA cohort.

highlighted in pancreatic cancer carcinogenesis and metastasis [8]. Our results are also supported by the suggestion that TMEM45B has important prognostic implications in tumor progression and metastasis. Moreover, the prognostic value of TMEM45B in primary carcinoma can be immediately applied to patients who undergo radical prostatectomy or biopsy, thus assisting in decision-making for subsequent treatment and follow-up planning [18].
Table 4.The association of TMEM45B expression and clinical characteristics of PCa in TCGA.

\begin{tabular}{|c|c|c|c|c|}
\hline Variable & Value & Mean & SD & p-value \\
\hline \multirow{2}{*}{$\begin{array}{l}\text { PSA } \\
\text { (Most Recent Results) }\end{array}$} & $\leq 10$ & -0.02 & 0.98 & \multirow{2}{*}{0.917} \\
\hline & $>10$ & 0.04 & 0.82 & \\
\hline \multirow{2}{*}{ T stage } & $\leq \mathrm{T} 2 \mathrm{C}$ & -0.23 & 0.74 & \multirow{2}{*}{$<0.001^{\rtimes}$} \\
\hline & $\geq \mathrm{T} 3 \mathrm{~A}$ & 0.11 & 1.09 & \\
\hline \multirow{2}{*}{ Gleason Score (RC) } & $\leq 7$ & -0.12 & 0.81 & \multirow{2}{*}{$0.007^{*}$} \\
\hline & $\geq 8$ & 0.12 & 1.16 & \\
\hline \multirow{2}{*}{$\mathrm{N}$ stage } & No & -0.03 & 1.00 & \multirow{2}{*}{$0.017^{*}$} \\
\hline & N1 & 0.27 & 1.14 & \\
\hline \multirow{2}{*}{ BCR } & positive & 0.10 & 1.12 & \multirow{2}{*}{0.477} \\
\hline & negative & -0.01 & 1001 & \\
\hline \multirow{2}{*}{$\begin{array}{l}\text { Recurrence/ } \\
\text { Progress }\end{array}$} & $\mathrm{Y}$ & 0.21 & 1.18 & \multirow{2}{*}{$0.014^{*}$} \\
\hline & $\mathrm{N}$ & -0.07 & 0.93 & \\
\hline \multirow{2}{*}{ Death } & $\mathrm{Y}$ & -0.09 & 0.71 & \multirow{2}{*}{0.826} \\
\hline & $\mathrm{N}$ & -0.02 & 0.98 & \\
\hline
\end{tabular}

The mechanism of TMEM45B promoting tumor progression and metastasis remains unclear. It is reported that TMEM45B down-regulation can arrest the cell cycle in the G1 phase to promote apoptosis. In contrast, apoptosis was induced when TMEM45B was over-expressed in pancreatic cell lines [8]. TMEM45B was also demonstrated to interact with the tubulointerstitial nephritisantigen (TINAG) involved in the FAK/PI3K/Akt-mediated apoptosis pathway in renal cell carcinoma. Moreover, TMEM45B is the miR-378, miR-422a and miR-483-5p co-target associated with organconfined diseases, and it determines the key hallmarks of non-small cell lung cancer [19]. The Paulo P' prostate cancer study showed that TMEM45B was the common target of the ERG and ETV ETS transcription factors which frequently participate in genomic rearrangements in prostate carcinomas and can contribute to tumorigenesis and progression through various pathways [20-22]. Downstream molecular targets are of utmost importance in understanding the mechanism of tumor biology, and the specific mechanism of ETS-TMEM45B-mediated tumor progression in PCa therefore requires further investigation.

\section{Conclusion}

The increased expression of TMEM45B is significantly associated with prostate carcinoma progression and metastasis, and this provides a new prognostic biomarker for predicting metastatic potential in PCa patients, and especially in Primary PCa patients.

Acknowledgments: This study was supported by National Natural Science Foundation of China (NSFC) [No. 81702534]. 


\section{References}

[1] SIEGEL RL, MILLER KD, JEMAL A. Cancer Statistics, 2017. CA Cancer J Clin 2017; 67: 7-30. https://doi.org/10.3322/ caac. 21387

[2] BATTY M, PUGH R, RATHINAM I, SIMMONDS J, WALKER E et al. The Role of alpha1-Adrenoceptor Antagonists in the Treatment of Prostate and Other Cancers. Int J Mol Sci 2016; 17: 1339. https://doi.org/10.3390/ijms17081339

[3] YOSHIMURA K, MINAMI T, NOZAWA M, KIMURA T, EGAWA $S$ et al. A Phase 2 Randomized Controlled Trial of Personalized Peptide Vaccine Immunotherapy with Lowdose Dexamethasone Versus Dexamethasone Alone in Chemotherapy-naive Castration-resistant Prostate Cancer. Eur Urol 2016; 70: 35-41. https://doi.org/10.1016/j.eururo.2015.12.050

[4] CHO H, HERZKA T, STAHLHUT C, WATRUD K, ROBINSON BD et al. Rapid in vivo validation of candidate drivers derived from the PTEN-mutant prostate metastasis genome. Methods 2015; 77-78: 197-204. https://doi.org/10.1016/j. ymeth.2014.12.022

[5] LIN D, WYATT A W, XUE H, WANG Y, DONG X et al. High fidelity patient-derived xenografts for accelerating prostate cancer discovery and drug development. Cancer Res 2014; 74: 1272-1283. https://doi.org/10.1158/0008-5472.CAN-132921-T

[6] LIN D, BAYANI J, WANG Y, SADAR MD, YOSHIMOTO M et al. Development of metastatic and non-metastatic tumor lines from a patient's prostate cancer specimen-identification of a small subpopulation with metastatic potential in the primary tumor. Prostate 2010; 70: 1636-1644. https://doi. org/10.1002/pros.21199

[7] OKADA N, YAMAMOTO T, WATANABE M, YOSHIMURA Y, OBANA E et al. Identification of TMEM45B as a protein clearly showing thermal aggregation in SDS-PAGE gels and dissection of its amino acid sequence responsible for this aggregation. Protein Expr Purif 2011; 77: 118-123. https:// doi.org/10.1016/j.pep.2011.01.011

[8] ZHAO LC, SHEN BY, DENG XX, CHEN H, ZHU ZG et al. TMEM45B promotes proliferation, invasion and migration and inhibits apoptosis in pancreatic cancer cells. Mol Biosyst 2016; 12: 1860-1870. https://doi.org/10.1039/c6mb00203j

[9] CUAJUNGCO MP, PODEVIN W, VALLURI VK, BUI Q, NGUYEN VH et al. Abnormal accumulation of human transmembrane (TMEM)-176A and $176 \mathrm{~B}$ proteins is associated with cancer pathology. Acta Histochem 2012; 114: 705-712. https://doi.org/10.1016/j.acthis.2011.12.006

[10] HAN MR, LONG J, CHOI JY, LOW SK, KWEON SS et al. Genome-wide association study in East Asians identifies two novel breast cancer susceptibility loci. Hum Mol Genet 2016; 25: 3361-3371. https://doi.org/10.1093/hmg/ddw164
[11] WRZESINSKI T, SZELAG M, CIESLIKOWSKI WA, IDA A, GILES R et al. Expression of pre-selected TMEMs with predicted ER localization as potential classifiers of ccRCC tumors. BMC Cancer 2015; 15: 518. https://doi.org/10.1186/ s12885-015-1530-4

[12] ZHANG Q, CHEN X, ZHANG X, ZHAN J, CHEN J. Knockdown of TMEM14A expression by RNAi inhibits the proliferation and invasion of human ovarian cancer cells. Biosci Rep 2016; 36: e00298. https://doi.org/10.1042/BSR20150258

[13] HU R, HU F, XIE X, WANG L, LI G et al. TMEM45B, upregulated in human lung cancer, enhances tumorigenicity of lung cancer cells. Tumour Biol 2016; 37: 12181-12191. https://doi.org/10.1007/s13277-016-5063-5

[14] TAYLOR BS, SCHULTZ N, HIERONYMUS H, GOPALAN A, XIAO Y et al. Integrative genomic profiling of human prostate cancer. Cancer Cell 2010; 18: 11-22. https://doi. org/10.1016/j.ccr.2010.05.026

[15] CANCER GENOME ATLAS RESEARCH NETWORK. The Molecular Taxonomy of Primary Prostate Cancer. Cell 2015; 163: 1011-1025. https://doi.org/10.1016/j.cell.2015.10.025

[16] CERAMI E, GAO J, DOGRUSOZ U, GROSS BE, SUMER SO et al. The cBio cancer genomics portal: an open platform for exploring multidimensional cancer genomics data. Cancer Discov 2012; 2: 401-404. https://doi.org/10.1158/2159-8290. CD-12-0095

[17] LONG J, CAI Q, SUNG H, SHI J, ZHANG B et al. Genomewide association study in east Asians identifies novel susceptibility loci for breast cancer. PLoS Genet 2012; 8: e1002532. https://doi.org/10.1371/journal.pgen.1002532

[18] BRUCE HM, STRICKER PD, GUPTA R, SAVDIE RR, HAYNES AM et al. Loss of AZGP1 as a Superior Predictor of Relapse in Margin-Positive Localized Prostate Cancer. Prostate 2016; 76: 1491-1500. https://doi.org/10.1002/pros.23233

[19] MOLINA-PINELO S, GUTIERREZ G, PASTOR MD, HERGUETA M, MORENO-BUENO G et al. MicroRNA-dependent regulation of transcription in non-small cell lung cancer. PLoS One 2014; 9: e90524. https://doi.org/10.1371/ journal.pone.0090524

[20] PAULO P, RIBEIRO FR, SANTOS J, MESQUITA D, ALMEIDA $M$ et al. Molecular subtyping of primary prostate cancer reveals specific and shared target genes of different ETS rearrangements. Neoplasia 2012; 14: 600-611.

[21] CLARK JP, COOPER CS. ETS gene fusions in prostate cancer. Nat Rev Urol 2009; 6: 429-439. https://doi.org/10.1038/ nrurol.2009.127

[22] HESSELS D, SCHALKEN JA. Recurrent gene fusions in prostate cancer: their clinical implications and uses. Curr Urol Rep 2013; 14: 214-222. https://doi.org/10.1007/s11934013-0321-1 